\title{
Tensorial Model for Photolithography Aerial Image Simulation
}

\author{
Caroline Fossati, Salah Bourennane, Romuald Sabatier, and Antonio Di Giacomo
}

Ecole Centrale Marseille Institut Fresnel, CNRS UMR 6133, 13397 Marseille Cedex 20, France

Correspondence should be addressed to Salah Bourennane, salah.bourennane@fresnel.fr

Received 21 July 2009; Accepted 31 August 2009

Recommended by Samir K. Mondal

In this paper, we propose to adapt the multilinear algebra tools to the tensor of Transmission Cross-Coefficients (TCC) values for aerial image simulation in order to keep the data tensor as a whole entity. This new approach implicitly extends the singular value decomposition (SVD) to tensors, that is, Higher Order SVD or TUCKER3 tensor decomposition which is used to obtain lower rank- $\left(K_{1}, K_{2}, K_{3}, K_{4}\right)$ tensor approximation (LRTA $\left.\left(K_{1}, K_{2}, K_{3}, K_{4}\right)\right)$. This model requires an Alternating Least Square (ALS) process known as TUCKALS3 algorithm. The needed number of kernels is estimated using two adapted criteria, well known in signal processing and information theory. For runtime improvement, we use the fixed point algorithm to calculate only the needed eigenvectors. This new approach leads to a fast and accurate algorithm to compute aerial images.

Copyright ( $) 2009$ Caroline Fossati et al. This is an open access article distributed under the Creative Commons Attribution License, which permits unrestricted use, distribution, and reproduction in any medium, provided the original work is properly cited.

\section{Introduction}

Over the last decades, photolithography has been instrumental to the historical trend, better known as Moore's law, of doubling chip density roughly every eighteen months while maintaining a nearly constant chip price. Going down to the $32 \mathrm{~nm}$ node, without changing the wavelength, we move closer and closer to the theoretical optical resolution limit. Therefore resolution Enhancement Techniques (RETs) have been developed in order to period all shape of an integrated circuit properly on the silicon wafer. One of the most wellknown RETs is the optical proximity correction (OPC), which consists of making mask pre-compensation of all nonlinear effects, optical diffraction, and interference effects. The reason behind correcting images distortions between the mask and the silicon due to this resolution limit. ModelBased Optical Proximity Correction (MBOPC) can treat layouts by deforming mask pattern to improve resolution on silicon. As for current masks, several billions of segments have to be moved during several iterations necessary to reach convergence; fast and accurate algorithms are mandatory to perform OPC on a mask in a reasonable time for industry.

To overcome this limitation, mathematical simplifications have to be done. As imaging with a lithography system (see Figure 1) is analogous to microscopy, the theory used in MBOPC is drawn from works originally designed for microscopy theory. Fourier optics was first used to describe the image formed by a microscope $[1,2]$. In 1951, Hopkins developed other formulation or method (1), that has been afterward used in other works involving image formation [3-6]. This formulation has the advantage of a four-way transmission function independent of the shape of the object to image. However, this method, in aerial image computation for photolithography, remains highly runtime consuming and needs a numerical approximation in order to reach production constraints.

In the solution called sum of coherent systems (SOCSs) provided by Cobb in [7], the Transfer Cross-Coefficients (TCCs) (2) are written in matrix format, then Singular Value Decomposition (SVD) that is used on this matrix, resulting in (3), allows to spare a huge amount of time while preserving a good accuracy as only the eigenvectors associated with the most energetic eigenvalues are used in calculus.

Clearly, we use Hopkins formulation, and a notation where $X$ will denote a tensor, $X$ a matrix, $x$ a vector, and $x$ a scalar.

The intensity image at the image plane is given by

$$
\begin{aligned}
I(u, v)= & \left(\frac{1}{2 \pi}\right)^{2} \sum_{i_{1}}^{I_{1}} \sum_{i_{2}}^{I_{2}} \sum_{i_{3}}^{I_{3}} \sum_{i_{4}}^{I_{4}} \mathcal{T} \mathcal{C C}\left(i_{1}, i_{2}, i_{3}, i_{4}\right) \\
& \times E\left(i_{1}, i_{2}\right) E^{*}\left(i_{3}, i_{4}\right) e^{i\left\{\left(i_{1}-i_{3}\right) u+\left(i_{2}-i_{4}\right) v\right\}},
\end{aligned}
$$




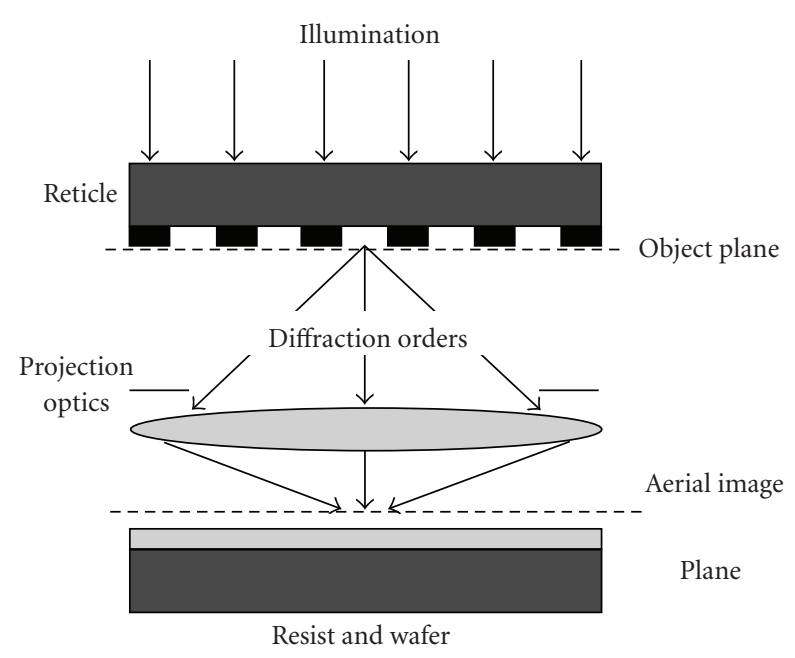

FIgURE 1: General optical lithography process diagram.

where

$$
\begin{aligned}
\mathcal{T C C}\left(i_{1}, i_{2}, i_{3}, i_{4}\right)= & 2 \pi \iint_{-\infty}^{+\infty} \Gamma(x, y) F\left(x+i_{1}, y+i_{2}\right) \\
& \times F^{*}\left(x+i_{3}, y+i_{4}\right) d x d y,
\end{aligned}
$$

and

$I($.$) intensity image at the image plane, E($.$) object being$ imaged (mask), $\Gamma($.$) mutual intensity function, describing$ coherence properties of the illumination, $F($.) coherent point spread function, describing properties of the projection system.

Typically, $I_{1}=I_{2}=I_{3}=I_{4}=I$ for nonastigmatic systems because of symmetry reasons. Those values are spatial frequencies, correspond to the range of values such that $\mathcal{T} \mathcal{C C}\left(i_{1}, i_{2}, i_{3}, i_{4}\right) \neq 0$, and depend on the optical system [8].

In his approach, Cobb [7] has unfolded tensor $\mathcal{T} \mathcal{C}$ to obtain a matrix $\mathrm{T}$ representation and then its eigendecomposition (SVD) leads to

$$
\mathbf{T}=\sum_{k=1}^{I^{2}} \lambda_{k} \mathbf{u}_{k} \mathbf{u}_{k}^{H}
$$

where the superscript " $H$ " denotes transpose conjugate. Then the lower rank matrix approximation of $\mathbf{T}$ gives the "kernels" used in aerial image calculation.

According to (2), the TCCs are depending on four indices, thus in this paper we propose to represent them as a four-way array. $I_{n}$ will denote the size of tensor $\mathcal{T} \mathcal{C}$ in dimension $n$. Tensor approach permits to treat a fourway array as a whole entity. In the considered problem, this approach allows to preserve interdimensional correlation avoiding possible data loss [9]. Low rank approximation, such as Singular Value Decomposition for two-dimensional array, considers significant and remaining parts of the data, they are based on data of most significant feature selection. The eigendecomposition of the matrix provides eigenvectors [10]. The $K_{n}$ eigenvectors associated with the $K_{n}$ largest (dominant) eigenvalues span the so-called "useful subspace." Useful subspace-based methods are applied to source localization in array processing and image denoising. These methods were adopted to four-way-array (tensor) [9]. The tensor model extends the classical matrix model [9]. This approach implicitly extends the SVD to tensors, that is, Higher Order SVD (HOSVD).

A fixed point algorithm comes then replacing HOSVD algorithm to spare some computational load, as already shown in [11].

The remainder of the paper is organized as follows In Section 2, some tensor definitions and properties are proposed. Section 3 proposes an original lower rank tensor approximation (LRTA) adapted to TCC data approximation. In Section 4, we propose a runtime improvement algorithm using an original method. After presenting some experimental results in Section 5, we conclude the paper in Section 6.

\section{Tensor Definitions and Properties}

In the following we remind some tensor definitions. For more details the reader can be referred to the papers in [12-14].

2.1. Tensor Definition. We consider an $N$-order tensor to represent an N-way array. Each of its elements is accessible through $N$ indices $\left(i_{1}, i_{2}, \ldots, i_{N}\right)$. This tensor is written $\mathcal{A} \in$ $\mathbb{C}^{I_{1} \times \cdots \times I_{N}}$, and each element $a_{i_{1}, \ldots, i_{N}}$. Each component is called an " $n$-mode" referring to the $n$th index of the tensor. A zeroorder tensor is a scalar, a first-order tensor is a vector, and a second-order tensor is a matrix.

To ease understanding this paper, some useful definitions are presented in the following.

2.2. Unfolding of Tensor $\mathcal{A}$. To study multiway data properties in a particular $n$-mode direction, as shown in Figure 2, we define the $n$-mode flattening matrix of tensor $\mathcal{A}$, written $\mathbf{A}_{n} \in \mathbb{C}^{I_{n} \times M_{n}}$, where

$$
M_{n}=I_{1} \cdots I_{n-1} I_{n+1} \cdots I_{N} \text {. }
$$

2.3. $n$-mode product $\times_{n}$. Consider tensor $\mathcal{A} \in \mathbb{C}^{I_{1} \times \cdots \times I_{N}}$ and a matrix $\mathbf{H} \in \mathbb{C}^{J \times I}$ with $I$ and $J \in \mathbb{N}^{*}$ (natural numbers excluding 0 ) and for all $n \in\{1, \ldots, N\}, n$-mode product between $\mathcal{A}$ and $\mathbf{H}$ is defined as

$$
\mathcal{B}=\mathcal{A} \times{ }_{n} \mathbf{H} \in \mathbb{C}^{I_{1} \times \cdots I_{n-1} \times J \times I_{n+1} \cdots \times I_{N}},
$$

Where $\times_{n}$ operator defines $n$-mode product. It generalizes matrix product to tensor and $n$-mode vector product in a particular n-mode.

Each element with index $\left(i_{1}, \ldots, i_{n-1}, j, i_{n+1}, \ldots, i_{N}\right)$ of tensor $\mathcal{B}$ is given by

$$
b_{i_{1} \ldots i_{n-1} j i_{n+1} \ldots i_{N}}=\sum_{i=1}^{I_{n}} a_{i_{1} \ldots i_{n-1} i i_{n+1} \ldots i_{N}} h_{j i} .
$$

2.4. Tensorial Scalar Product. Tensorial scalar product is defined as follows. Suppose two $N$ order tensors: $\mathcal{A}$ and 

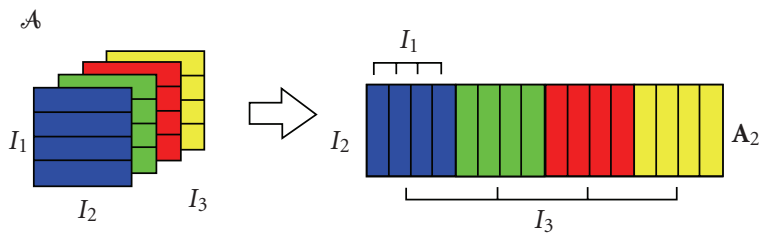

FIgURe 2: Flattened matrix $\mathbf{A}_{2} \in \mathbb{R}^{I_{2} \times I_{3} I_{1}}$ in 2-mode of tensor $\mathcal{A} \in$ $\mathbb{R}^{I_{1} \times I_{2} \times I_{3}}$.

$\mathscr{B} \in \mathbb{C}^{I_{1} \times \cdots \times I_{N}}$. Scalar product between $\mathcal{A}$ and $\mathcal{B}$ is given by

$$
\langle\mathcal{A B}\rangle=\sum_{i_{1}, \ldots, i_{N}} a_{i_{1}, \ldots, i_{N}} b_{i_{1}, \ldots, i_{N}}
$$

2.5. Useful Subspace Definition. Let us define $E^{(n)}$ the $n$ thmode vector space of dimension $I_{n}$, associated with the $n$ thmode of tensor $\mathcal{A}$. By definition, $E^{(n)}$ is generated by the column vectors of the $n$ th-mode flattening matrix. The $n$ thmode flattening matrix $\mathbf{A}_{n}$ of tensor $\mathcal{A} \in \mathbb{C}^{I_{1} \times \cdots \times I_{N}}$ is defined as a matrix from $\mathbb{C}^{I_{n} \times M_{n}}$, with

$$
M_{n}=I_{1} \cdots I_{n-1} I_{n+1} \cdots I_{N}
$$

$\mathbf{A}_{n}$ columns are the $I_{n}$-dimensional vectors obtained from $\mathcal{A}$ by varying index $i_{n}$ and keeping the other indices fixed. In $\mathbf{A}_{n}$, the $I_{n}$-dimensional vectors obtained from $\mathcal{A}$ are ordered with respect to their index for each mode but the $n$th mode. These vectors are called in the following the $n$ th-mode vectors of tensor $\mathcal{A}$. The lower rank- $\left(K_{1}, K_{2}, \ldots, K_{N}\right)$ tensor $\mathcal{A}$ approximation is possible by extending the classical subspace approach to tensors by assuming that, whatever the $n$ thmode vector space of dimension $I_{n}, E^{(n)}$ is the superposition of two orthogonal subspaces:

(i) the useful subspace $E_{1}^{(n)}$ of dimension $K_{n} . E_{1}^{(n)}$ is generated by the $K_{n}$ eigenvectors of matrix $\mathbf{A}_{n}$, which are associated with the $K_{n}$ largest eigenvalues;

(ii) and the subspace $E_{2}^{(n)}$ of dimension $I_{n}-K_{n} \cdot E_{2}^{(n)}$ is generated by the $I_{n}-K_{n}$ singular vectors of matrix $\mathbf{A}_{n}$, which are associated with the $I_{n}-K_{n}$ smallest eigenvalues,

such that $E^{(n)}=E_{1}^{(n)} \oplus E_{2}^{(n)}$. The dimensions $K_{1}, K_{2}, \ldots, K_{N}$ can be estimated by means of the well-known (Akaike Information Criterion) AIC or (Minimum Description Length) MDL criteria [10]. These are entropy-based Information Criteria which have had a fundamental impact in statistical model evaluation problems.

2.6. Frobenius Norm of a Tensor. Frobenius norm of a tensor $\mathcal{A}$, written $\|\mathcal{A}\|$, is given by

$$
\|\mathcal{A}\|=\langle\mathcal{A} \mathcal{A}\rangle=\sum_{i_{1}, \ldots, i_{N}} a_{i_{1}, \ldots, i_{N}}^{2} .
$$

2.7. External Product. External product "。” of several vectors $\mathbf{e}^{(1)}, \ldots, \mathbf{e}^{(N)}$ belonging, respectively, to $E^{(1)}, \ldots, E^{(N)}$, of dimension $I_{1}, \ldots, I_{N}$ defines a tensor $\mathcal{A} \in \mathbb{C}^{I_{1} \times \ldots \times I_{N}}$ of rank 1 :

$$
\mathcal{A}=\mathbf{e}^{(1)} \circ \mathbf{e}^{(2)} \circ \cdots \circ \mathbf{e}^{(N)}
$$

in such a way that whatever the indices $\left(i_{1}, \ldots, i_{N}\right)$, the element $a_{i_{1} \ldots i_{N}}$ of tensor $\mathcal{A}$ is defined by product $a_{i_{1} \ldots i_{N}}=$ $\prod_{n=1}^{N} e_{i_{n}}^{(n)}$, where $e_{i_{n}}^{(n)}$ is the $i_{n}^{\text {th }}$ component of vector $\mathbf{e}^{(n)}$.

2.8. Tensor Rank. In case of matrices, rank plays an important role in SVD, in canonical decomposition and in lower rank approximation. Consider the definitions of a tensor rank

Classical Rank. Consider tensor of order $N, \mathcal{A} \in \mathbb{C}^{I_{1} \times \cdots \times I_{N}}$. From II-A, tensor $\mathcal{A}$ is built on $N$ vector space: $E^{(1)}, \ldots, E^{(N)}$, of dimension $I_{1}, \ldots, I_{N}$. Tensor $\mathcal{A}$ has rank one if, for all $n=$ $1, \ldots, N, N$ vectors $\mathbf{u}^{(n)} \in E^{(n)}$ exist, such as $\mathcal{A}$ is equal to their external product:

$$
\mathcal{A}=\mathbf{u}^{(1)} \circ \cdots \circ \mathbf{u}^{(N)} .
$$

By extension, $\mathcal{A}$ is a tensor of $\operatorname{rank} K$, if $K$ is the minimal number or tensor of rank 1 which, by summation, give $\mathcal{A}$. We write the rank of tensor $\mathcal{A}$ by $\operatorname{Rank}(\mathcal{A})=K$.

$n$-Mode Rank. It is possible to define $n$-mode rank of a tensor as the generalization of column vectors lines vectors rank of a matrix. $n$-mode rank of a tensor $\mathcal{A} \in \mathbb{C}^{I_{1} \times \cdots \times I_{N}}$, written $\operatorname{Rank}_{n}(\mathcal{A})$, is the rank of flattened matrix $\mathbf{A}_{n}$ of $\mathcal{A}$ in $n$-mode, $\operatorname{Rank}_{n}(\mathcal{A})=\operatorname{Rank}\left(\mathbf{A}_{n}\right)$.

$\operatorname{Rank}-\left(K_{1}, \ldots, K_{N}\right)$. A tensor $\mathcal{A} \in \mathbb{C}^{I_{1} \times \cdots \times I_{N}}$ has rank$\left(K_{1}, \ldots, K_{N}\right)$ if, for all $n=1, \ldots, N, \operatorname{Rank}_{n}(\mathcal{A})=K_{n}$.

\section{Tensor Approximation Based on Lower Rank Tensor Approximation (LRTA) Algorithm}

The main objective of this paper is to adopt the multilinear algebra tools to approximate the tensor $\mathcal{T} \mathcal{C}$ for system transmission based on Lower Rank Tensor Approximation (LRTA) algorithm; we will consider in the rest of the paper $N=4$ which corresponds to the fourth-order tensor of the TCC values, $\mathcal{T} \mathcal{C C} \in \mathbb{C}^{I_{1} \times I_{2} \times I_{3} \times I_{4}}$. The corresponding $n$-mode rank values are denoted $K_{1}, K_{2}, K_{3}, K_{4}$. We assume that the value of $K_{n}$ is known or is estimated (see Section 4.2 to know how to estimate them) for all $n=1$ to 4 .

As an extension to the vector and matrix cases, in the tensor formulation, the projectors on the $n$ th-mode vector spaces are determined by computing the rank$\left(K_{1}, K_{2}, K_{3}, K_{4}\right)$ approximation of $\mathcal{T} \mathcal{C C}$ in the least-squares sense. From a mathematical point of view, the rank$\left(K_{1}, K_{2}, K_{3}, K_{4}\right)$ approximation of $\mathcal{T} \mathcal{C} \mathcal{C}$ is represented by tensor $\widehat{\mathcal{T C C}}$ which minimizes the quadratic tensor Frobenius norm $\|\mathcal{T} \mathcal{C C}-\widehat{\mathcal{T C C}}\|^{2}$ subject to the constraint that $\widehat{\mathcal{T C C}} \in \mathbb{C}^{I_{1} \times I_{2} \times I_{3} \times I_{4}}$ is a $\operatorname{rank}-\left(K_{1}, K_{2}, K_{3}, K_{4}\right)$ tensor. The 
(1) Input: data tensor $\mathcal{T} \mathcal{C} \mathcal{C}$, and dimensions $K_{1}, K_{2}, K_{3}, K_{4}$ of all $n$-mode signal subspaces.

(2) Initialization $k=0$ : For $n=1$ to 4 , calculate the projectors $\mathbf{P}_{0}^{(n)}$ given by HOSVD- $\left(K_{1}, K_{2}, K_{3}, K_{4}\right)$ :

(a) $n$-mode unfold $\mathcal{T} \mathcal{C} \mathcal{C}$ into matrix $\mathbf{T C C}_{n}$;

(b) Compute the SVD of TCC $_{n}$;

(c) Compute matrix $\mathbf{U}_{0}^{(n)}$ formed by the $K_{n}$ eigenvectors associated with the $K_{n}$ largest singular values of $\mathrm{TCC}_{n} . \mathbf{U}_{0}^{(n)}$ is the initial matrix of the $n$-mode signal subspace orthogonal basis vectors;

(d) Form the initial orthogonal projector $\mathbf{P}_{0}^{(n)}=\mathbf{U}_{0}^{(n)} \mathbf{U}_{0}^{(n)^{T}}$ on the $n$-mode signal subspace;

(e) Compute the HOSVD- $\left(K_{1}, K_{2}, K_{3}, K_{4}\right)$ of tensor $\mathcal{T} \mathcal{C C}$ given by $\mathscr{B}_{0}=\mathcal{T} \mathcal{C} \mathcal{C} \times{ }_{1} \mathbf{P}_{0}^{(1)} \times{ }_{2} \cdots \times{ }_{4} \mathbf{P}_{0}^{(4)}$

(3) ALS loop: Repeat until convergence, that is, for example, while $\left\|\mathscr{B}_{k+1}-\mathscr{B}_{k}\right\|^{2}>\varepsilon, \varepsilon>0$ being a prior fixed threshold,

(a) For $n=1$ to 4 :

(i) Form $\mathscr{B}^{(n), k}: \mathscr{B}^{(n), k}=\mathcal{T} \mathcal{C C} \times{ }_{1} \mathbf{P}_{k+1}^{(1)} \times{ }_{2} \mathbf{P}_{k+1}^{(2)} \times{ }_{3} \mathbf{P}_{k+1}^{(3)} \times{ }_{4} \mathbf{P}_{k+1}^{(4)}$;

(ii) $n$-mode unfold tensor $\mathscr{B}^{(n), k}$ into matrix $\mathbf{B}_{n}^{(n), k}$;

(iii) Compute matrix $\mathbf{C}^{(n), k}=\mathbf{B}_{n}^{(n), k} \mathbf{T C C}_{n}^{T}$;

(iv) Compute matrix $\mathbf{U}_{k+1}^{(n)}$ composed of the $K_{n}$ eigenvectors associated with the $K_{n}$ largest eigenvalues of $\mathbf{C}^{(n), k} \cdot \mathbf{U}_{k}^{(n)}$ is the matrix of the $n$-mode signal subspace orthogonal basis vectors at the $k$ th iteration;

(v) Compute $\mathbf{P}_{k+1}^{(n)}=\mathbf{U}_{k+1}^{(n)} \mathbf{U}_{k+1}^{(n)}$;

(b) Compute $\mathscr{B}_{k+1}=\mathcal{T} \mathcal{C} \mathcal{C} \times{ }_{1} \mathbf{P}_{k+1}^{(1)} \times{ }_{2} \cdots \times{ }_{4} \mathbf{P}_{k+1}^{(4)}$;

(c) Increment $k$.

(4) Output: the estimated signal tensor is obtained through $\widehat{\mathcal{T C C}}=\mathcal{T} \mathcal{C C} \times{ }_{1} \mathbf{P}_{k_{\text {stop }}}^{(1)} \times_{2} \cdots \times_{4} \mathbf{P}_{k_{\text {stop }}}^{(4)} . \widehat{\mathcal{T C C}}$ is the rank- $\left(K_{1}, K_{2}, K_{3}, K_{4}\right)$ approximation of $\mathcal{T} \mathcal{C} \mathcal{C}$, where $k_{\text {stop }}$ is the index of the last iteration after the convergence of TUCKALS3 algorithm.

Algorithm 1: Lower rank- $\left(K_{1}, K_{2}, K_{3}, K_{4}\right)$ tensor approximation (LRTA- $\left.\left(K_{1}, K_{2}, K_{3}, K_{4}\right)\right)$.

TUCKER3 tensor decomposition also known as HigherOrder SVD (HOSVD) [15] and lower rank- $\left(K_{1}, K_{2}, K_{3}, K_{4}\right)$ tensor approximation (LRTA- $\left.\left(K_{1}, K_{2}, K_{3}, K_{4}\right)\right)$ has recently been used as multimode (Principal Component Analysis) PCA, in seismic for wave separation based on a useful subspace method, in image processing for face recognition and expression analysis and noise filtering of color images. Tensor $\widehat{\mathcal{T C C}}$ can be expressed with the TUCKER3 model [12] as

$$
\widehat{\mathcal{T C C}}=\mathscr{D} \times{ }_{1} \mathbf{U}^{(1)} \times{ }_{2} \mathbf{U}^{(2)} \times{ }_{3} \mathbf{U}^{(3)} \times{ }_{4} \mathbf{U}^{(4)} .
$$

The least square solution implies that

$$
\mathcal{D}=\mathcal{T} \mathcal{C} \mathcal{C} \times{ }_{1} \mathbf{U}^{(1) T} \times{ }_{2} \mathbf{U}^{(2) T} \times{ }_{3} \mathbf{U}^{(3) T} \times{ }_{4} \mathbf{U}^{(4) T} .
$$

Thus the best lower approximation of tensor $\mathcal{T} \mathcal{C C}$ denoted $\widehat{\mathcal{T C C}}$ is to orthogonally project, for every $n$-mode, the vectors of tensor $\mathcal{T} \mathcal{C} \mathcal{C}$ on the $n$ th-mode useful subspace $E_{1}^{(n)}$, for all $n=1$ to $4[9,13]$

$$
\widehat{\mathcal{T C C}}=\mathcal{T} \mathcal{C C} \times{ }_{1} \mathbf{P}^{(1)} \times{ }_{2} \mathbf{P}^{(2)} \times{ }_{3} \mathbf{P}^{(3)} \times{ }_{4} \mathbf{P}^{(4)} .
$$

In (14), $\mathbf{P}^{(n)}=\mathbf{U}^{(n)} \mathbf{U}^{(n) T}$ is called the projector of the $n$ th-mode space. The estimation of the projectors is a difficult nonlinear least square problem that is generally solved owing to an ALS algorithm referred to as TUCKALS3 algorithm $[12,16]$.

TUCKALS3 algorithm $[12,16]$ is an optimal algorithm to estimate the different $n$-mode useful subspaces. The description of TUCKALS3 algorithm, used in rank- $\left(K_{1}, K_{2}, K_{3}, K_{4}\right)$ approximation, is provided in Algorithm 1.
In this algorithm, the second-order statistics comes from the SVD of matrix $\mathbf{T C C}_{n}$ at step $2 \mathrm{~b}$, which is equivalent, up to $1 / M_{n}$ multiplicative factor, to the estimation of tensor $\mathcal{T} \mathcal{C}$-mode vectors [9]. The definition of $M_{n}$ is given in (8). In the same way, at step 3 (a)iii, matrix $\mathbf{C}^{(n), k}$ is, up to $1 / M_{n}$ multiplicative factor, the estimation of the covariance matrix between tensor $\mathcal{T} \mathcal{C} \mathcal{C}$ and tensor $\mathscr{B}^{(n), k} n$ mode vectors. According to step $3(\mathrm{a}) \mathrm{i}, \mathcal{B}^{(n), k}$ represents data tensor $\mathcal{T} \mathcal{C C}$ filtered in every $m$ th-mode but the $n$ th-mode, by projection-filters $\mathbf{P}_{l}^{(m)}$, with $m \neq n, l=k$ if $m>n$ and $l=$ $k+1$ if $m<n$. TUCKALS3 algorithm has recently been used to process a multimode PCA in order to perform white noise removal in color images [9]. A good approximation of the rank- $\left(K_{1}, K_{2}, K_{3}, K_{4}\right)$ approximation can simply be achieved by computing the HOSVD- $\left(K_{1}, K_{2}, K_{3}, K_{4}\right)$ of tensor $\mathcal{T} \mathcal{C}$ $[9,15]$. Indeed, the HOSVD- $\left(K_{1}, K_{2}, K_{3}, K_{4}\right)$ of $\mathcal{T} \mathcal{C} \mathcal{C}$ consists of the initialization step of TUCKALS3 algorithm, and hence can be considered as a suboptimal solution for the rank- $\left(K_{1}, K_{2}, K_{3}, K_{4}\right)$ approximation of tensor $\mathcal{T} \mathcal{C}$ [15]. This HOSVD-based technique has recently been used in [9] for image denoising.

\section{Runtime Improvement: Fixed Point Algorithm}

As computing LRTA involves to compute four times Singular Value Decomposition for $\mathcal{T}$ C C decomposition, we propose a fixed point algorithm based on Gram-Schmidt orthogonalisation to lower runtime. 
(1) Choose $K$, the number of principal axes or eigenvectors required to estimate. Consider matrix $\mathbf{T}$ and set $p \leftarrow 1$.

(2) Initialize eigenvector $\mathbf{u}_{p}$ of size $d \times 1$, for example, randomly;

(3) Update $\mathbf{u}_{p}$ as $\mathbf{u}_{p} \leftarrow \mathbf{T} \mathbf{u}_{p}$;

(4) Do the Gram-Schmidt orthogonalization process $\mathbf{u}_{p} \leftarrow \mathbf{u}_{p}-\sum_{j=1}^{j=p-1}\left(\mathbf{u}_{p}^{T} \mathbf{u}_{j}\right) \mathbf{u}_{j}$;

(5) Normalize $\mathbf{u}_{p}$ by dividing it by its norm: $\mathbf{u}_{p} \leftarrow \mathbf{u}_{p} /\left\|\mathbf{u}_{p}\right\|$.

(6) If $\mathbf{u}_{p}$ has not converged, go back to step 3.

(7) Increment counter $p \leftarrow p+1$ and go to step 2 until $p$ equals $K$.

Algorithm 2: Fixed-point.

4.1. Fixed Point Algorithm. We propose to adapt a Fixed Point algorithm [17] to replace SVD in LRTA [11], Algorithm 1, it allows to spare a high amount of computational load while preserving a high level of accuracy and has never been used in optical domain yet. Although SVD needs to be computed only once, this method is very useful during model tuning as several variants are compiled.

One way to compute the $K$ orthonormal basis vectors is to use Gram-Schmidt method (Algorithm 2).

The eigenvector with dominant eigenvalue will be measured first. Similarly, all the remaining $K-1$ basis vectors (orthonormal to the previously measured basis vectors) will be measured one by one in a reducing order of dominance. The previously measured $(p-1)$ th basis vectors will be used to find the $p^{\text {th }}$ basis vector. The algorithm for $p$ th basis vector will converge when the new value $\mathbf{u}_{p}^{+}$and old value $\mathbf{u}_{p}$ are such that $\mathbf{u}_{p}^{+T} \mathbf{u}_{p}=1$. It is usually economical to use a finite tolerance error to satisfy the convergence criterion $\left\|\mathbf{u}_{p}^{+T} \mathbf{u}_{p}-1\right\|<\eta$, where $\eta$ is a prior fixed threshold.

Let $\mathbf{U}=\left[\mathbf{u}_{1}, \mathbf{u}_{2}, \ldots, \mathbf{u}_{K}\right]$ be the matrix whose columns are the $K$ orthonormal basis vectors. Then $\mathbf{U U}^{T}$ is the projector onto the subspace spanned by the $K$ eigenvectors associated with the $\mathrm{K}$ largest eigenvalues. This subspace is also called "signal subspace." It can be used in LRTA- $\left(K_{1}, K_{2}, K_{3}, K_{4}\right)$ to retrieve the basis vectors $\mathbf{U}_{0}^{(n)}$ in step $2 \mathrm{c}$ of Algorithm 1. Thus, the initialization step is faster since it does not need the $I_{n}$ basis vectors but only the $K_{n}$ first ones and it does not need the step $2 \mathrm{~b}$, that is SVD of the data tensor $n$-mode unfolding matrix TCC $_{n}$.

According to what have been explained before, fixed point algorithm will replace SVD in LRTA algorithm, in order to improve runtime. However, a prerequisite to this algorithm is the knowledge of each $n$-mode rank. A method to determine them is developed in next subsection.

4.2. n-Mode Rank Estimation. Whereas a common way to obtain $K_{1}, K_{2}, K_{3}, K_{4}$ values is to use empirical data [18], we wish to present here a scientific tool optimizing the calculation. Each projector $\mathbf{P}^{(n)}, n=1,2,3,4$, is estimated by the truncation of unfolding matrices TCC $_{n}$ using SVD, that is, by keeping the $K_{n}$ eigenvectors associated with the $K_{n}$ largest singular values of $\mathbf{T C C}_{n}, n=1,2,3,4$. In order to estimate the value of $K_{n}$ for each $n$-mode, we extend the wellknown detection criteria [10]. These criteria, initially, are developed for estimating the number of largest eigenvalues of any matrix. Thus, the optimal signal subspace dimension is obtained merely by minimizing one of (Akaike's Information Criterion) AIC [19] or (Minimum Description Length) MDL [20] criteria. This approach does note require any subjective threshold settings. The number of eigenvectors can be determined from the eigenvalues of the $n$-mode unfolding matrix TCC $_{n}$. Thus, this method presents the advantage of being self-consistent as it computes the optimal number of kernels for any illumination configuration.

Consequently, for each $n$-mode unfolding of $\mathcal{T C C}$, the form of detection criterion AIC can be expressed as

$$
\operatorname{AIC}(k)=-2\left(I_{n}-k\right) M_{n} \ln \frac{g\left(\lambda_{k+1}, \ldots, \lambda_{I_{n}}\right)}{\mathcal{A}\left(\lambda_{k+1}, \ldots, \lambda_{I_{n}}\right)}+2 k\left(2 I_{n}-k\right),
$$

and the MDL criterion is given by

$$
\begin{aligned}
\operatorname{MDL}(k)= & -\left(I_{n}-k\right) M_{n} \ln \frac{g\left(\lambda_{k+1}, \ldots, \lambda_{I_{n}}\right)}{\mathcal{A}\left(\lambda_{k+1}, \ldots, \lambda_{I_{n}}\right)} \\
& +\frac{1}{2} k\left(2 I_{n}-k\right) \ln M_{n},
\end{aligned}
$$

where $\mathcal{G}$ and $\mathcal{A}$ are geometricand arithmetic mean, respectively. $\left(\lambda_{i}\right)_{1 \leq i \leq I_{n}}$ are $I_{n}$ eigenvalues of the data matrix of the $n$-mode unfolding $\mathcal{T} \mathcal{C} \mathcal{C}: \lambda_{1} \geq \lambda_{2} \geq \cdots \geq \lambda_{I_{n}}$, and $M_{n}$ is the number of columns of the $n$-mode unfolding $\mathcal{T} \mathcal{C}$.

The $n$-mode rank $K_{n}$ is the value of $k\left(k=1, \ldots, I_{n}-1\right)$ which minimizes AIC or MDL criterion.

\section{Experimental Results}

The proposed methods can be applied to any four-way array of data set such as image, multicomponent seismic signals, hyperspectral images.

We wish to compare LRTA algorithm benefits over SOCS algorithm. We use square size matrices with increasing rows and columns number as data set to compare algorithms runtime and accuracy.

5.1. Runtime Improvement. We first focus on Fixed Point algorithm runtime gain toward SVD algorithm. Figure 3 shows runtime curves of increasing size matrices decomposition. Matlab SVD algorithm is used. We have to compute about 10 eigenvectors for each matrix with fixed point 
TABLE 1: Some numerical values corresponding with Figure 3.

\begin{tabular}{|c|c|c|c|c|c|c|}
\hline Matrix size & 120 & 720 & 1320 & 1920 & 2520 & 3000 \\
\hline SVD runtime (sec) & 0.109 & 6.262 & 38.911 & 122.810 & 279.762 & 489.808 \\
\hline Fixed point runtime (sec) & 0.010 & 0.406 & 1.762 & 4.536 & 9.220 & 14.522 \\
\hline SVD/FP rate & 10.629 & 15.441 & 22.083 & 27.073 & 30.343 & 33.728 \\
\hline
\end{tabular}

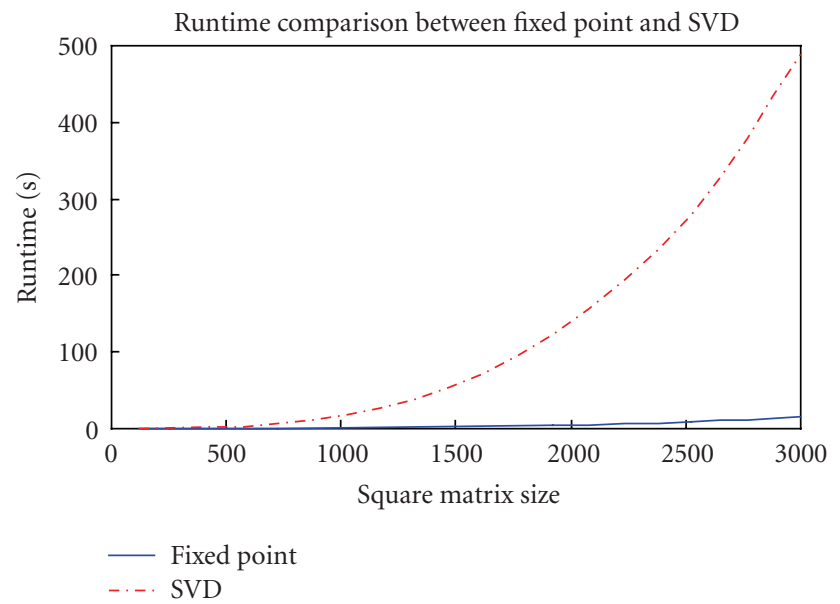

Figure 3: Computational times (in sec.) as a function of rows and columns number.

algorithm. This value is provided by AIC criterion and corresponds to an average value of eigenvectors number used in classical OPC models. However, as fixed point algorithm runtime is linear with eigenvectors number to compute, Table 1 shows that this number can be increased up to around 300 eigenvectors for $3000 \times 3000$ matrices and up to around 100 eigenvectors for $120 \times 120$ matrices.

The computations have been run with Matlab on a 2.4 GHz dual core Pentium with 4Go RAM under Windows $\mathrm{XP}$.

5.2. AIC and MDL Criteria. Figure 4(a) shows AIC and MDL functions of a fourth-order tensor filled with random data. The minimum value in AIC and MDL curves gives rank estimation value of considered mode. Figure 4(b) shows the normalized energy of the eigenvalues of the same array. Those curves are helpful to understand that the rank given by AIC and MDL criteria is really connected to the eigenvectors associated with the largest eigenvalues.

5.3. Fixed Point Algorithm Reconstruction Error. In order to assess our algorithm precision toward SVD decomposition, Figure 5 shows reconstruction error of both fixed point and SVD algorithms, $\left\|\mathbf{T}-\mathbf{T}^{\prime}\right\| /\|\mathbf{T}\|$, where $\mathbf{T}$ denotes the original matrix and $\mathbf{T}^{\prime}$ the reconstructed matrix. For constancy purposes with fixed point algorithm, the same number of eigenvectors is used for both algorithms. It is difficult to distinguish the two curves on Figure 5; however fixed point algorithm reconstruction error remains very slightly above SVD reconstruction error.
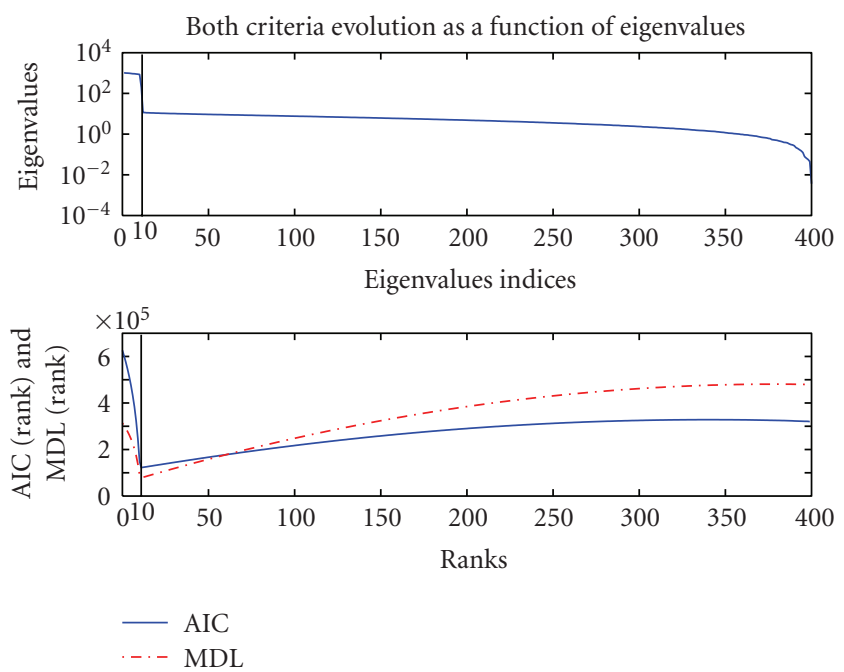

(a) AIC and MDL estimation criteria

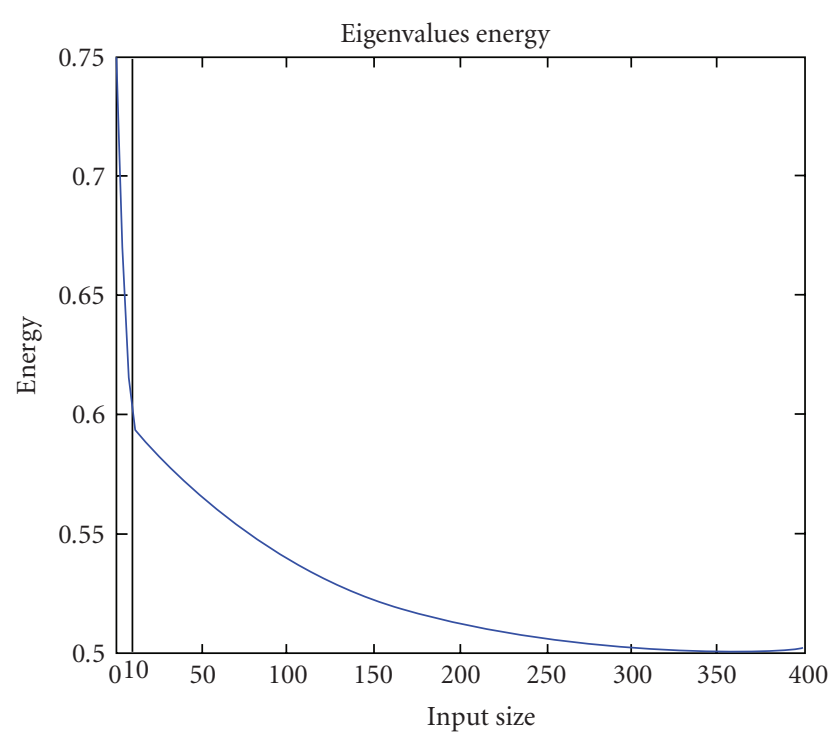

(b) Normalized energy of matrix eigenvalues

FIGURE 4: Energy of matrix eigenvalues and comparison between $\mathrm{AIC}$ and MDL criteria.

5.4. Comparison between LRTA and SOCS Algorithms. Figure 6 shows a comparison of SOCS and LRTA algorithms reconstruction error and both algorithms runtime.

To obtain these curves, random tensors of increasing size have been created. They have been unfolded following Cobb's 


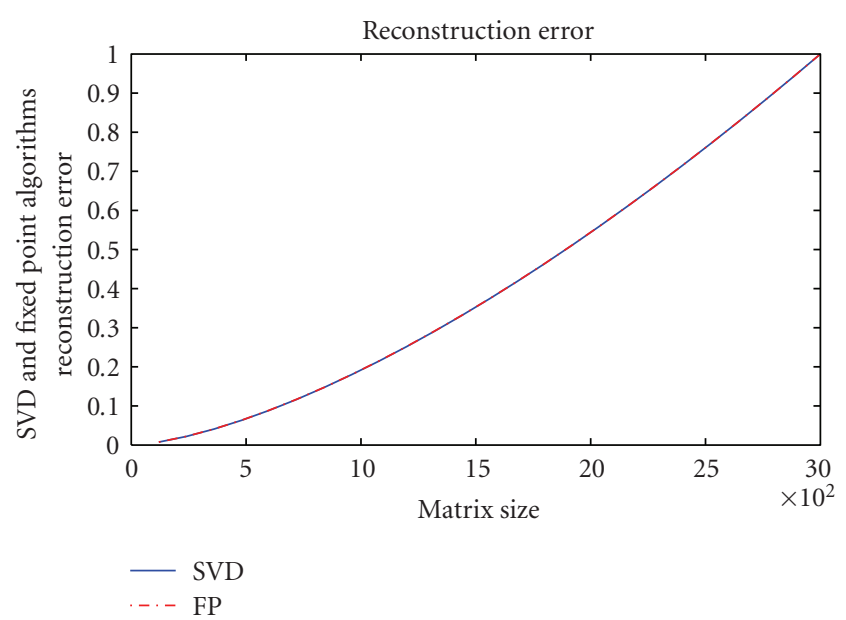

Figure 5: Reconstruction error comparison between SVD and Fixed point algorithm.

method [7], decomposed using SVD algorithm, recomposed using only the dominant eigenvectors.

In the same time, they have been decomposed using LRTA algorithm with fixed point and also recomposed using only the dominant eigenvectors.

In both cases, the error function is the same than used in Section 5.3 to compute fixed point algorithm versus SVD algorithm error.

The curves show clearly the benefit of our method based on tensor data representation and on multilinear algebra tools.

The computations have been run with Matlab on a 2.4 GHz dual core Pentium with 4Go RAM under Windows $\mathrm{XP}$.

5.5. Comparison of Aerial Images. Figure 7 shows aerial images computed using Full Hopkins equation and with SOCS algorithm and LRTA algorithm, for 4 (Figure 7(a)), 10 (Figure 7(b)) and 20 kernels (Figure 7(c)). The structure used is a T-shaped geometry of total size $720 \mathrm{~nm} \times 720 \mathrm{~nm}$. The model used corresponds to a $193 \mathrm{~nm}$ wavelength illumination, $0.75 \mathrm{NA}$ and $0.6 \sigma$, optical diameter is $1280 \mathrm{~nm}$, and grid size is $1 \mathrm{~nm}$.

A lower number of kernels are sufficient for the LRTA algorithm to give a result close to the full hopkins. This is confirmed by the obtained results shown in Figure 8 using the same calculus only with 4 kernels for L-shaped geometry. Note that this sufficient number is estimated using AIC criterion.

Considering Full Hopkins gives the aerial image as close as possible to the image really obtained on the wafer. One can see that the aerial image obtained using LRTA approximation better looks like real image than one obtained by SOCS algorithm.

\section{Conclusion}

We have proposed here a new approach to Transfer CrossCoefficients data set approximation in diffraction theory of

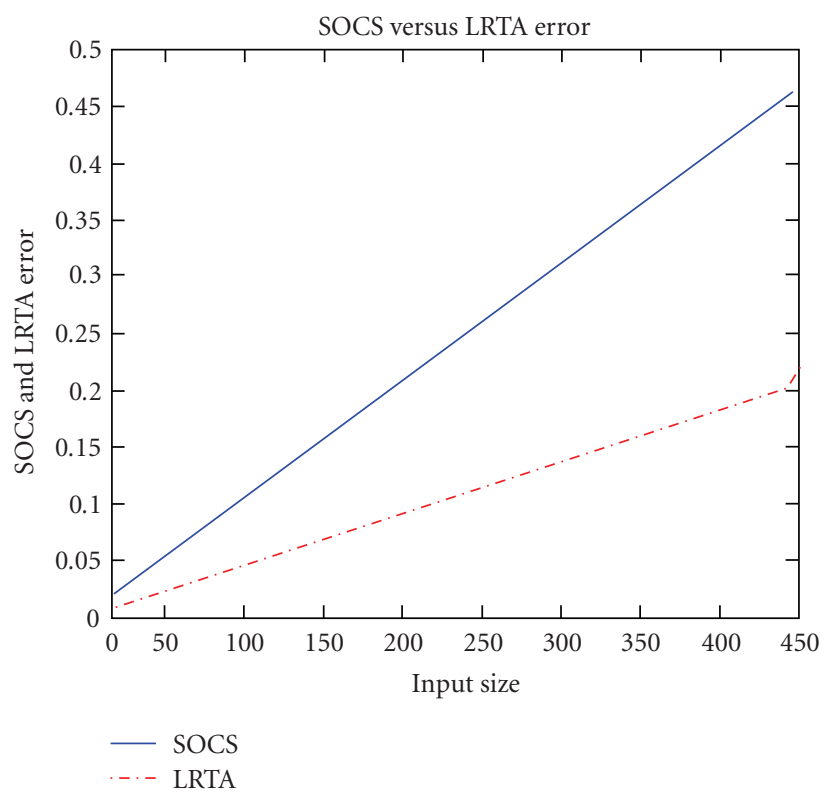

(a) Comparison of LRTA and SOCS reconstruction error

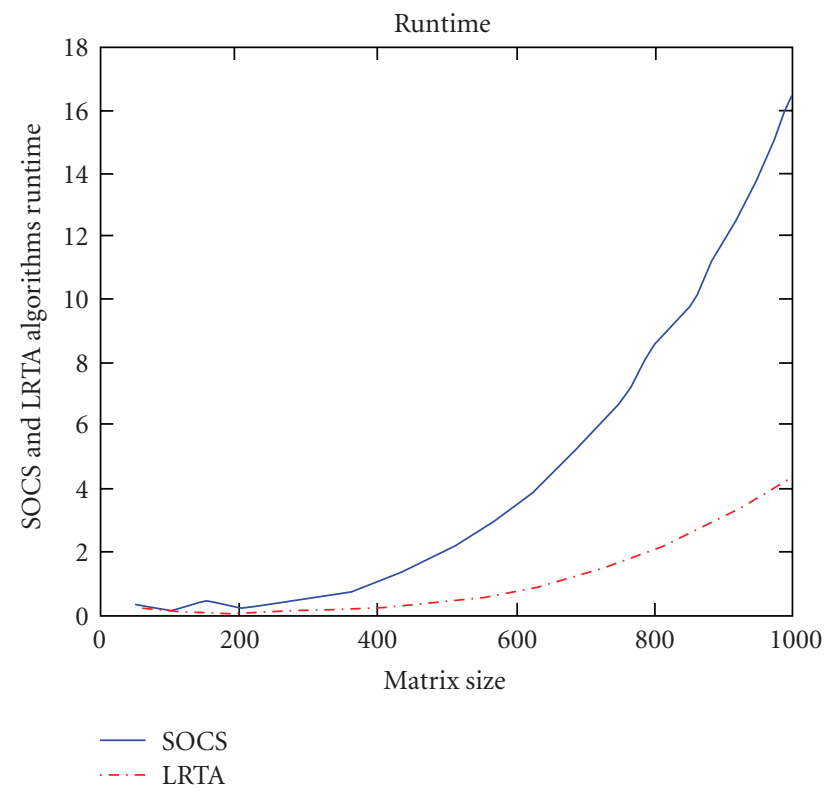

(b) Comparison between SOCS and LRTA runtime

Figure 6: Comparison between LRTA and SOCS.

optical images based on multilinear algebra tools. The goal of this work is two way axed, it allows to adapt complex physical equations to tensor computation and to improve runtime by using fixed point algorithm instead of HOSVD while preserving accuracy by using LRTA and AIC algorithm. We have proven our method to be faster and more accurate than existing SOCS method, as we obtain a lower reconstruction error than SOCS algorithm, whatever the size of input data is. Those methods have proven their efficiency in other domains, such as four-way images restoration or denoising 


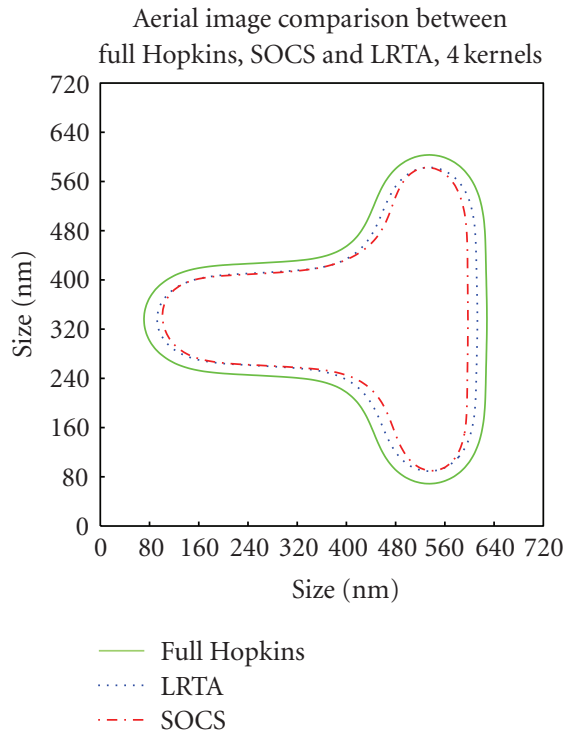

(a)

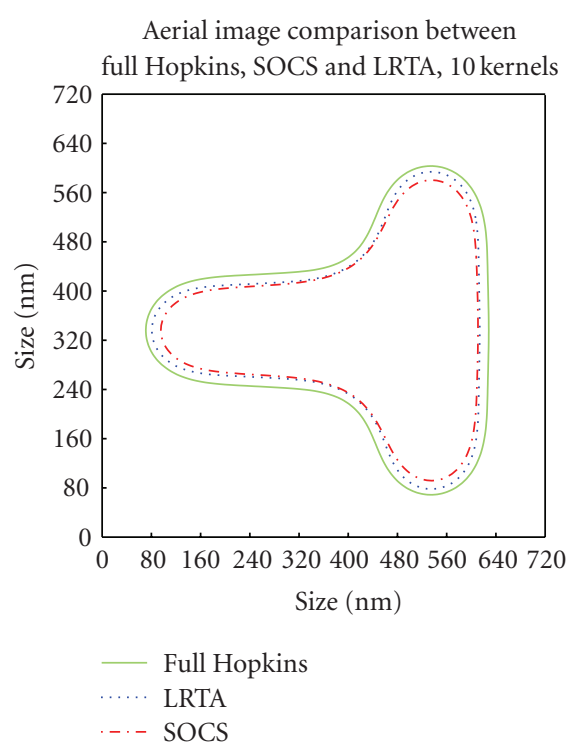

(b)

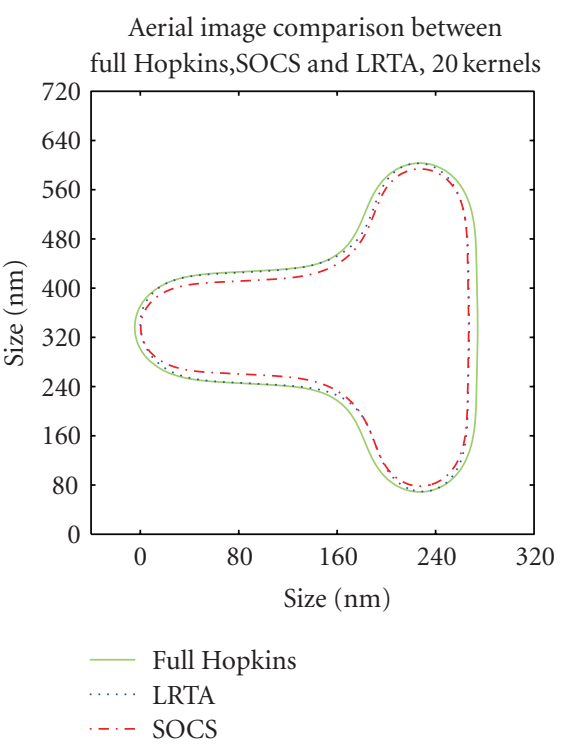

(c)

Figure 7: Aerial image comparison between SOCS, LRTA, and full Hopkins.

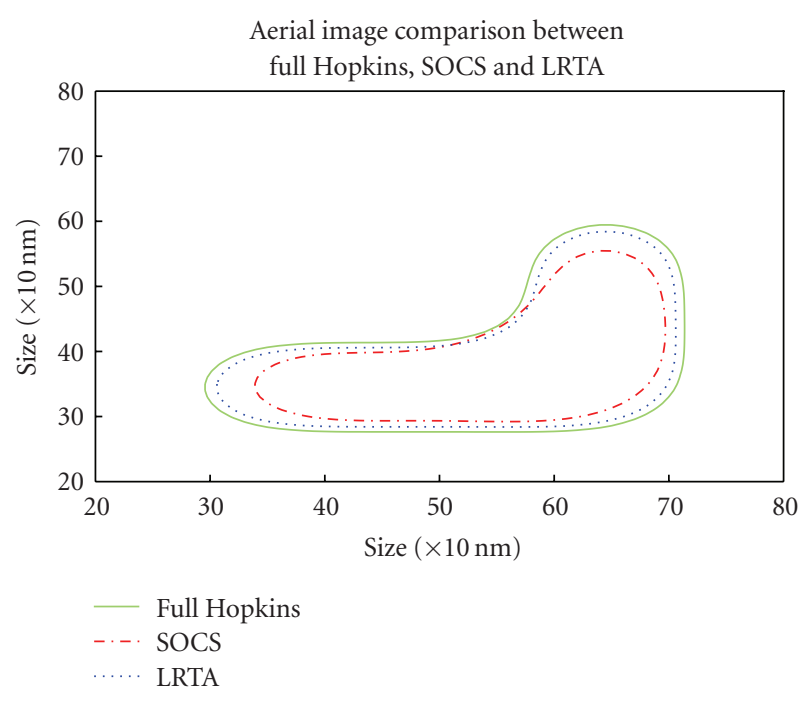

FIGURE 8: Aerial image comparison between SOCS, LRTA, and full Hopkins.

and for wave separation of multicomponent seismic data, but have never been implemented in imaging theory yet. In further work, we will bring more detailed experimental data to express in real cases the extent of the improvement of our method.

\section{Acknowledgment}

The authors would like to thank the anonymous reviewers for their careful reading and their fruitful remarks, which have contributed in improving the quality of the paper.

\section{References}

[1] E. Hecht, Optics, Addison-Wesley, Reading, Mass, USA, 1987.

[2] J. Goodman, Introduction to Fourier Optics, McGraw-Hill, New York, NY, USA, 1996.

[3] H. Hopkins, "The concept of partial coherence in optics," Proceedings of the Royal Society A, vol. 208, pp. 263-277, 1951.

[4] H. Hopkins, "On the diffraction theory of optical images," Proceedings of the Royal Society A, vol. 217, no. 1131, pp. 408432, 1952.

[5] M. Born and E. Wolf, Principles of Optics, Pergamon Press, London, UK, 6th edition, 1980.

[6] P. Flanner, "Two-dimensional optical imaging for photolithography simulation,” Tech. Rep. UCB ERL M86 57, Electronics Research Laboratory, University of California, Berkeley, Calif, USA, July 1986.

[7] N. Cobb, Fast optical and process proximity correction algorithms for integrated circuit manufacturing, Ph.D. thesis, University of California, Berkeley, Calif, USA, 1998.

[8] D. Newmark, "Computer aided design tools for phase-shift masks and spatial filtering," Tech. Rep. UCB ERL M91 117, Electronics Research Laboratory, University of California, Berkeley, Calif, USA, December 1991.

[9] D. Muti and S. Bourennane, "Survey on tensor signal algebraic filtering," Signal Processing, vol. 87, pp. 237-249, 2007.

[10] M. Wax and T. Kailath, "Detection of signals by information theoretic criteria," IEEE Transactions on Acoustics, Speech, and Signal Processing, vol. 33, no. 2, pp. 387-392, 1985.

[11] R. Sabatier, C. Fossati, S. Bourennane, and A. Di Giacomo, "Fast approximation of transfer cross coefficient for optical proximity correction," Optics Express, vol. 16, no. 19, pp. 15249-15253, 2008.

[12] H. A. L. Kiers, "Towards a standardized notation and terminology in multiway analysis," Journal of Chemometrics, vol. 14, no. 3, pp. 105-122, 2000. 
[13] L. De Lathauwer and J. Castaing, "Tensor-based techniques for the blind separation of DS-CDMA signals," Signal Processing, vol. 87, no. 2, pp. 322-336, 2007.

[14] M. Vasilescu and D. Terzopoulos, "Multilinear independent components analysis," in Proceedings of the IEEE Computer Society Conference on Computer Vision and Pattern Recognition (CVPR '05), vol. 1, pp. 547-553, San Diego, Calif, USA, June 2005.

[15] L. De Lathauwer, B. De Moor, and J. Vandewalle, "A multilinear singular value decomposition," SIAM Journal on Matrix Analysis and Applications, vol. 21, pp. 1253-1278, 2000.

[16] N. D. Sidiropoulos and R. Bro, "On the uniqueness of multilinear decomposition of N-way arrays," Journal of Chemometrics, vol. 14, no. 3, pp. 229-239, 2000.

[17] A. Hyvärinen and E. Oja, "A fast-fixed point algorithm for independent component analysis," Neural Computation, vol. 9, no. 7, pp. 1483-1492, 1997.

[18] C. Zuniga and E. Tejnil, "Heuristics for truncating the number of optical kernels in Hopkins image calculations for model-based OPC treatment," in Optical Microlithography XX, Proceedings of SPIE, San Jose, Calif, USA, February 2007.

[19] H. Akaike, "A new look at the statistical model identification," IEEE Transactions on Automatic Control, vol. 19, no. 6, pp. 716-723, 1974.

[20] J. Rissanen, "A universal prior for integers and estimation by minimum description length," The Annals of Statistics, vol. 11, no. 2, pp. 416-431, 1983. 

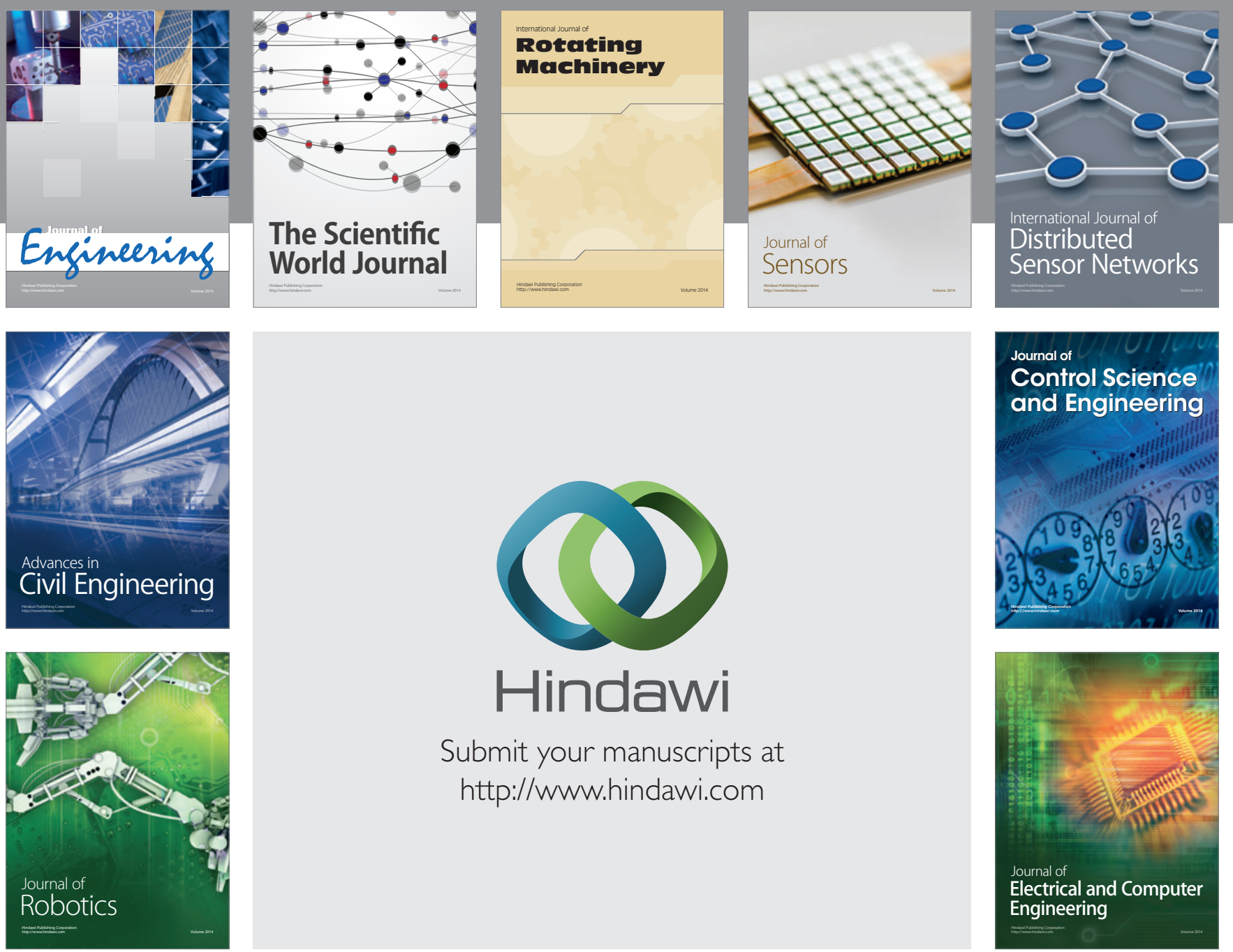

Submit your manuscripts at

http://www.hindawi.com
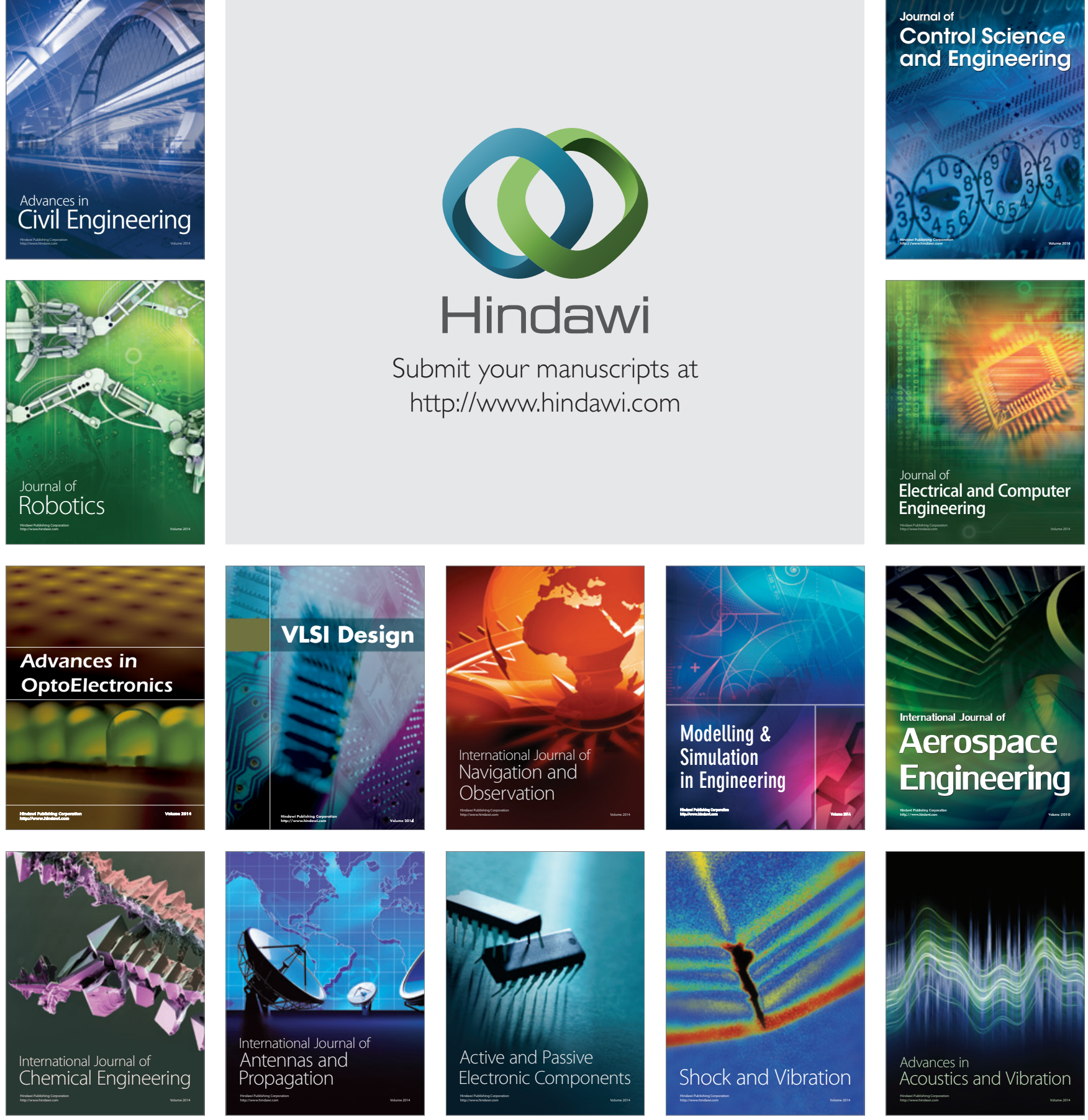